\title{
Radiation Damage in Macromolecular Crystallography
}

${ }^{a}$ Elspeth F. Garman and ${ }^{b}$ Martin Weik

${ }^{a}$ Laboratory of Molecular Biophysics, Department of Biochemistry, University of Oxford, South Parks Road, Oxford OX1 3QU, UK; ${ }^{b}$ Institut de Biologie Structurale, Univ. Grenoble Alpes, CEA, CNRS, F-38044 Grenoble, France

E-mail: elspeth.garman@bioch.ox.ac.uk and weik@ibs.fr

\begin{abstract}
Radiation damage inflicted on macromolecular crystals during X-ray diffraction experiments remains a limiting factor for structure solution, even when samples are cooled to cryotemperatures $(\sim 100 \mathrm{~K})$. Efforts to establish mitigation strategies are ongoing and various approaches, summarised below, have been investigated over the last 15 years, resulting in a deeper understanding of the physical and chemical factors affecting damage rates. The recent advent of X-ray free electron lasers permits 'diffraction before destruction' by providing highly brilliant and short (a few tens of fs) X-ray pulses. New $4^{\text {th }}$ generation synchrotron sources now coming on line with higher X-ray flux densities than those available from $3^{\text {rd }}$ generation synchrotrons, will bring the issue of radiation damage once more to the fore for structural biologists.
\end{abstract}

Keywords X-ray-matter interactions, global and specific radiation damage, radicals and their scavengers, absorbed dose, radiation damage mitigation, cryocrystallography

Running head Radiation damage 


\section{Introduction}

Since the earliest days of macromolecular crystallography (MX), radiation damage to the sample during X-ray irradiation has been a limiting factor for three-dimensional structure solution. At room temperature (RT), the highest resolution reflections from diffracting protein crystals typically start to fade before a full dataset has been collected. For RT experiments, Blundell and Johnson [1] recommended that when the intensity of a monitored reflection dropped to 0.85 of its original value $\left(I_{0}\right)$, the crystal should be replaced by a new one, but if the crystal supply was limited, this could be pushed to $0.7 I_{0}$. Following the pioneering work of Hope on flash cooling of ribosome crystals [2] and the introduction of the loop mounting method for samples [3], the development of cryocrystallographic techniques in the 1990s [47] allowed routine flash cooling of crystals to $\sim 100 \mathrm{~K}$. At such cryotemperatures it was found that radiation damage rates could be reduced by around a factor of 70 compared with those seen at RT [8]. However, in 1994, reflection fading was again observable during quantitative experiments at cryotemperatures with a white beam carried out at the Daresbury synchrotron [9]. With the advent of higher flux density X-ray beams produced by $3^{\text {rd }}$ generation synchrotrons in the mid to late $1990 \mathrm{~s}$, damage effects were observed at $100 \mathrm{~K}$ with monochromatic beams. Below we summarise some of the ongoing efforts over the last 15 years to elucidate the manifestations and origins of radiation damage and to establish mitigation strategies using various approaches. These have resulted in a deeper understanding of the physical and chemical factors affecting damage rates. However, new $4^{\text {th }}$ generation synchrotron sources now coming on-line with even higher flux densities than hitherto utilised, will bring the issue of radiation damage into even sharper focus for structural biologists. An awareness of the effects of radiation damage on diffraction data and on the macromolecular structures derived from them, will therefore become increasingly important. The use of very short X-ray pulses (a few tens of fs) produced by X-ray free electron laser sources allows 'diffraction before destruction' with no significant radiation damage. The new methodology associated with these experiments is covered elsewhere in this book in the chapter by Chapman.

\section{Interaction of X-ray photons with matter}

The physics involved in radiation damage is well understood and characterized, although the same cannot be said about the chemical aspects. When an X-ray beam of the energy range usually used for MX (6-15 keV) is incident on a sample, it interacts by three main mechanisms (Figure 1): elastic (Thomson, coherent) scattering, Compton (incoherent) 
scattering, and photoelectric absorption [10]. Most of the beam passes straight through the sample without interacting at all: for instance for a $12.4 \mathrm{keV}(1 \AA)$ beam and a $30 \mu \mathrm{m}[100$ $\mu \mathrm{m}]$ thick crystal, $99.2 \%$ [98\%] of the photons will transit unaffected and be captured by the beamstop (this reduces to $87.3 \%$ [64\%] for a $5 \mathrm{keV}$ beam). Of the interacting $0.8 \%$ [2\%] of the beam, $0.06 \%[0.16 \%]$ will undergo elastic scattering which is the desired interaction for diffraction: the photon scatters from the electrons in the crystal without leaving any energy behind. However, due to the cross sections subtended by the atoms to the X-rays, $92 \%$ [92\%] of the interacting photons $(\sim 0.74 \%$ [1.84\%] of the incident beam) are absorbed, and the photons lose some or all of their energy in the sample.

At $12.4 \mathrm{keV}$, Compton scattering accounts for around $9 \%$ of these absorption events, and involves the inelastic collision of an X-ray photon with the electrons in an atom to give a lower energy X-ray photon. This photon may or may not escape the sample, depending on how much energy was lost on the collision and the size and composition of the crystal. The third interaction, photoelectron production, accounts for the rest of the absorption events and is the most prevalent process that is deleterious to the integrity of the sample. Here the incident X-ray photon is completely absorbed by the electron, which is then ejected, carrying with it the energy of the incoming photon minus the binding energy of that electron when in the atom. The X-ray photoelectron cross sections for inner shell electrons are significant, and rise steeply with atomic number. The resulting primary 'photoelectron' has a diffusion range of several microns at $100 \mathrm{~K}$ (e.g., $18.7 \mathrm{keV}$ photons have a range of 3 to $4 \mu \mathrm{m}$ in protein crystals [11]). It loses its energy by excitation and ionization of atoms in its path until it eventually thermalises, producing many secondary electrons with lower energy as it goes. A photoelectron produced by a $12.4 \mathrm{keV}$ photon has enough energy to cause up to $\sim 500$ further ionisations, assuming $25 \mathrm{eV}$ is required for each ionisation [12]. These events may occur directly in the protein, including its primary shell of hydration (direct events), or in the solvent channels of the crystal (indirect events).

The atom which originally absorbed the incident photon and ejected the photoelectron can decay in two different ways: either by Auger electron emission, whereby an outer shell electron is ejected, carrying with it the energy released as an electron in a lower shell drops down to take the vacant place left by the photoelectron in the inner shell; or by fluorescent Xray emission, whereby the emitted X-ray photon carries away the energy difference between the electronic shells when an outer-shell electron falls inwards. This fluorescent X-ray may be absorbed or may escape, depending on its energy and the sample size. The probability of 
fluorescent emission is very low for light elements but increases with atomic number, and for iron ${ }^{55} \mathrm{Fe}$ it is $30 \%$.

Any absorption event will potentially cause energy to be lost in the crystal and thus results in radiation damage to the constituent molecules.

Note that the precise classifications of primary and secondary radiation damage differs between scientific fields; here 'primary' refers to the initial photoelectron, and 'secondary' to the products it induces. In addition, in the case of crystalline material, tertiary damage is suffered by the lattice which may be destabilised by damage to atoms involved in crystal contacts, or by gas formation within the crystal.

From a chemical viewpoint, the ionisations described above will lead to electron-gain and electron-loss (holes) centres resulting from the direct effects and giving rise to radicals. These may recombine, leading to an excited state, the deactivation of which may or may not cause damage. These recombination processes compete with charge separation through migration, which for electron and hole centres may occur by tunnelling (essentially temperature independent) or hopping (temperature dependent) to given sites in the protein, where the radicals become localized.

\section{Mitigation of secondary radiation damage by cryocooling}

The primary absorption events (Compton and photoelectric effects) described above are a fact of physics: they cannot be avoided in the diffraction experiment and are temperature independent. However, diffusion of most of the secondary radiation induced products can be prevented by cooling the sample to around $100 \mathrm{~K}$ during data collection. This temperature was originally selected as it can be reached conveniently using open flow nitrogen cryostats [13], but it was a fortuitous choice, since below $110 \mathrm{~K}$, hydroxyl radicals produced by the radiolysis of water are thought to be immobile [14]. At $100 \mathrm{~K}$, all larger species produced by reactions of secondary products, such as peroxide and superoxide (in the presence of oxygen), are unable to diffuse through the solvent channels of the crystal. However, even at very low temperatures, electrons and holes are both able to migrate by quantum mechanical tunneling [15], which is a temperature independent phenomenon. In addition, any thermal energy available will allow them to 'hop', but the probability of this phenomenon is temperature dependent. The effect of these mobile species at $100 \mathrm{~K}$ is to induce specific structural damage to the protein (see below). Prompt hole transfer to the protein from primary absorption events occurring in solvent adjacent to the protein might also be expected. 
The overall effect of cryocooling is to significantly improve the dose tolerance of macromolecular crystals at $100 \mathrm{~K}$ so that for most samples the rate of radiation damage is decreased by nearly two orders of magnitude. The use of cryocrystallographic techniques has thus allowed $3^{\text {rd }}$ generation synchrotron beams to be used to good effect to solve macromolecular crystal structures; $84 \%$ of the crystal structures deposited in the PDB with temperature of data collection information have been determined with the crystal held at or near $100 \mathrm{~K}(90 \%$ held below $160 \mathrm{~K})$. In particular, the success of the Multi-wavelength Anomalous Diffraction (MAD) method of structure solution has relied on taking data at two or more different wavelengths from the same crystal, a feat possible at $100 \mathrm{~K}$ but not at RT.

\section{Absorbed dose, estimating dose and dose limits}

To monitor the effects of radiation damage, various observables can be plotted as a function of image number or time. However, using either of these parameters as the abscissa has the disadvantage that experiments performed under different conditions cannot be compared. Thus a much more generally applicable metric is required for the study and quantitation of radiation damage effects.

The absorbed dose is such a metric: it is the energy absorbed per mass of sample, expressed in $\mathrm{J} / \mathrm{kg}=$ gray or Gy units. This is not a directly measurable quantity, but must be estimated from both the contents of the crystal and the particular experimental conditions. For a sample with linear absorption coefficient $\mu_{a b s}$, the incident beam intensity, $J_{0}$, falls off exponentially (first order decay) with crystal thickness, $x$, to become $J$, according to the relationship:

$$
J=J_{0} e^{-\mu_{a b s} x}
$$

The energy lost in the sample is then $J_{0}-J$ multiplied by the incident photon energy. The cross section subtended by the sample to the X-ray beam, $\mu_{a b s}$, is computed from its atomic composition, which includes the amino acid residues of the protein, any bound heavy atoms, ligands or nucleotides, and also the components of the buffer in the solvent channels. The cross section for the photoelectric effect rises steeply with atomic number, $Z$, and the presence of a few heavy atoms in the unit cell can have a large effect on the absorption of X-rays and thus the absorbed dose. Also required for the dose calculation are the beam characteristics, namely its energy, size and profile, the incident photon flux $\left(J_{0}\right)$, and the exposure time. This requires regular calibration of synchrotron beamlines and straightforward methodology has been developed to expedite this step [16]. 
As a general dose yard stick, $1 \mathrm{MGy} \mathrm{s}^{-1}$ will be absorbed by a $100 \mu \mathrm{m}$ cubed metal-free crystal in a $100 \mu \mathrm{m} \times 100 \mu \mathrm{m}$ beam of $12.4 \mathrm{keV}(1 \AA)$ X-rays and a flux of $10^{13}$ photons s${ }^{-1}$, and will cause approximately 1 ionisation per 20 amino acid residues per second [12].

In order to facilitate dose estimation in a spatially and temporally resolved way for MX, the computer program RADDOSE-3D [17] has been developed, which can model an X-ray diffraction experiment. It includes the ability to model complicated data collection protocols [18] such as helical scans [19] and translations, as well as MAD experiments. An experimentally measured beam profile can be used to gain a more realistic photon flux distribution, and buffer components can be entered as $\mathrm{mM}$ concentrations. The program can also use polygon crystal shapes to improve the dose estimation. Its shortcomings are that account is not taken of the escape of fluorescent X-rays (produced by heavy atoms after photoelectron ejection - see above), and also that the likely escape of the primary photoelectron [20] is neglected. Since the proportion of electrons escaping becomes significant for crystals with volumes smaller than $20 \mu^{3}$, the doses computed by RADDOSE-3D for micron-sized crystals are vastly overestimated.

The earlier RADDOSE versions 1, 2 and 3 [21-23] were developed when crystals were generally smaller than beams and thus crystal rotation could be neglected. These versions gave the maximum dose experienced by the crystal (usually at its centre - see below) and this approach was widely used prior to the availability of RADDOSE-3D.

The distribution of damage in an irradiated crystal depends pivotally on the beam profile. If this is top-hat shaped, the crystal will be uniformly irradiated, and will be damaged at the same rate throughout. If, however, the flux is distributed in a rough Gaussian profile, the centre of a crystal which is aligned in the beam and rotated on an axis intersecting the peak of the beam, will suffer faster damage than the parts farther away and on the tails of the flux distribution (Figure 2). A study of the damage rates in 43 datasets from 34 different crystals correlating beam profile with data quality showed that the beam profile was a vital parameter required to explain the results [24].

It is clear that it is hard to quote a representative dose for a crystal such as the one shown in Figure 2a, where the values range from zero up to $112 \mathrm{MGy}$. The options include the average dose for the whole crystal or the maximum dose. To address this problem, a new metric, Diffraction Weighted Dose (DWD), has been introduced, and is computed by RADDOSE-3D. It combines information from the aggregation of dose within each volume element of the 
crystal up to a given time, with the way the crystal is being exposed at that moment. DWD has been experimentally validated by using three very different sized beams and comparing the resulting data characteristics plotted against DWD. In the same study, using DWD to plan the experiment, it was also shown that spreading dose through sample more evenly improves data quality, in this case achieved by offsetting the rotation axis from the beam centre [25].

For a biological sample, there is a limit to the level of radiation it can tolerate without losing its integrity. Henderson [26] suggested, by analogy and using observations of the diffraction lifetime of 2D crystals in electron microscopy at $77 \mathrm{~K}$, an approximate dose limit for MX, $D_{1 / 2}$, after which half the total diffraction intensity $\left(0.5 I_{0}\right)$ would have disappeared. The 'Henderson limit' is $20 \mathrm{MGy}$, and has been a useful yardstick for planning experiments. It is important to note that a crystal might not survive until the limit is reached (e.g. if there were susceptible residues at crystal contacts [27]).

The $D_{1 / 2}$ for MX was experimentally measured at $100 \mathrm{~K}$ to be $43 \mathrm{MGy}$ [28] using apo- (no iron) and holo-ferritin (one iron atom for every two amino acid residues, leading to $\mu_{a b s}$ that is more than double that of the apo-protein enzyme for X-rays at $12.4 \mathrm{keV}$ ). However, it was found that at $D_{1 / 2}$, the electron density maps were very significantly affected by specific structural damage (see below), and thus a limit of $30 \mathrm{MGy}$, corresponding to $0.7 I_{0}$, was recommended in order to arrive at biologically meaningful structures. Interestingly and serendipitously, the $0.7 I_{0}$ limit is the same as recommended by Blundell and Johnson [1] for RT data collection.

The $100 \mathrm{~K}$ MX dose limit of $30 \mathrm{MGy}$ was determined for summed intensity data to $2.2 \AA$ resolution, but since the higher resolution reflections fade fastest, a resolution dependent limit of $10 \mathrm{MGy} \AA^{-1}$ has been proposed [29], i.e. after an absorbed dose of $10 \mathrm{MGy}$, a $2 \AA$ diffraction pattern would fade to $3 \AA$. Note that at RT there appears to be a large range of doses (from a few kGy to $1.5 \mathrm{MGy}$ ) for $0.7 I_{0}$ tolerated by protein crystals, but $150 \mathrm{kGy}$ has recently been suggested as a suitable RT limit [30].

\section{Global radiation damage at cryo temperatures}

The effects of radiation damage which are observed in reciprocal space are together termed 'global' damage. The most visible sign as the absorbed dose increases is the gradual fading of the intensity of the diffraction pattern, with the highest-resolution reflections being the first to disappear [9]. Thus the resolution limit of the data is degraded. Once the data are processed, other effects become clear from the inspection of the cell parameters and merging statistics: 
the unit cell volume increases with dose [31-33], Wilson $B$-factors increase, merging $R$ factors worsen, $I / \sigma(I)$ decreases as the intensity decreases and the noise $(\sigma(I))$ concomitantly increases, and the mosaicity usually increases with dose.

The expansion of unit cell with dose was originally thought to be a possible metric that could be used at the beam line during the experiment to judge whether a crystal should be discarded due to excessive radiation damage [31]. However, systematic experimental studies of the phenomenon led to the conclusion that the rate of cell volume increase was too variable, even among fragments of the same large crystal, for it to be a robust metric [34, 35]. The volume expansion is thought to be caused by hydrogen gas produced from radiolytic reactions, which gathers at domain boundaries [36] causing the unit cell to increase in size.

There are three indications of global radiation damage that can be usefully plotted as a function of absorbed dose, $D$ (Figure 3). The first is the summed intensity of a dataset or data wedge $\left(I_{n}\right)$ divided by the initial summed intensity of that dataset or wedge $\left(I_{1}\right), I_{n} / I_{1}[28]$. This is a preferred representation of the intensity decay over $I / \sigma(I)$, since $\sigma(I)$ increases with dose. The second informative plot is of the relative scaling $B$-factor, $B_{r e l}$, which is the difference in Wilson $B$-factor between the $n$th dataset or wedge and that of the first dataset or wedge. This function has been observed to rise linearly with dose and its gradient gives an indication of the radiation sensitivity of the crystal. This coefficient of sensitivity is defined as $s_{A D}=\Delta B_{\text {rel }} / 8 \pi^{2} \Delta D$ and has a value for hen egg white lysozyme (HEWL) crystals at $100 \mathrm{~K}$ of $0.012 \AA^{2} /$ Gy. It does not appear to vary significantly with protein species [37]. The third indicator that is sometimes used is a pairwise $R$-factor, $R_{d}$, between identical and symmetry related reflections occurring on different diffraction images, plotted against the difference in dose $\Delta D$ between those images [38].

Unfortunately these three indicators can give inconsistent results for analysis of the same data (see for instance plots of $I_{n} / I_{l}$ and $R_{d}$ in Figs. 3 and 4 of [39]). This is an as yet unresolved issue in systematic radiation damage studies.

\section{Specific structural radiation damage at cryotemperatures}

X-ray irradiation produces specific structural and chemical damage besides the global radiation damage that affects the diffraction power described above. Disulfide bridges were already identified as being particularly radiation-sensitive at RT as early as 1988 [40]. When highly brilliant $3^{\text {rd }}$ generation synchrotron sources came online in the mid-1990s, systematic studies at cryotemperatures identified changes in the electron density of disulfide bridges and 
glutamic and aspartic acid residues (Figure 4) as being the first obvious signs of specific radiation damage, originating from reduction and decarboxylation, respectively [31, 32, 41$]$. Interestingly, chemically identical species appeared not to be equally affected, indicating that specific damage is not caused by X-ray absorption of the susceptible atom or group (primary damage), but is rather the consequence of interaction with secondary radicals generated by the primary event $[33,34]$ (the primary event typically being a result of the photoelectric effect, see above [27]). Disulfide bridges, for example, trap a secondary electron and then either elongate upon formation of a disulfide radical anion [42] or break following a complex multitrack model [43]. Among the residues suffering specific damage, those located in protein active sites have consistently been observed as being most susceptible (e.g. in acetylcholinesterase [41], bacteriorhodopsin [44], photoactive yellow protein [45], DNA photolyase [46], malate dehydrogenases [47], carbonic anhydrase [48] and fluorescent proteins [49]), possibly because they are subject to chemical and geometric strains [31, 50]. Consequently, the biological information extracted from protein crystal structures can be altered by convolution with radiation-induced structural and chemical modifications.

Although there is now a wide body of literature devoted to understanding the mechanisms behind the specific damage of proteins, far less is known regarding radiation-induced damage to crystalline nucleic acids and the wider class of nucleoprotein complexes. Recent systematic studies on a protein-DNA complex [51] and a $91 \mathrm{kDa}$ RNA-protein complex [52] have clearly shown both DNA and RNA to be much more robust in terms of specific structural damage than protein.

The specific structural damage inflicted by X-rays can also be put to good use in so-called radiation-induced phasing (RIP) [53]. Two low-dose data sets are separated by an X-ray burn of several MGy that generates the specific damage exploited for phasing as in the single isomorphous replacement (SIR) method [53, 54]. RIP can be combined with single wavelength anomalous diffraction (SAD) [55] and specific damage created by UV light instead of X-rays (UV-RIP) [56].

Note that the issue mentioned above concerning non-top-hat beam profiles also affects the differential specific damage rates, and these result in a mixture of molecular conformations across the crystal (e.g. disulfide bonds in various stages of breakage). Thus the resulting electron density maps will be an average over these states, and will not be as sharp as when a top-hat profile beam is used. The unit cell distribution will also be affected, those in the centre expanding faster than those at the margins of the crystal. This causes non-isomorphism both 
within single datasets and between datasets during MAD experiments. The reflection intensity changes induced by these non-isomorphism effects can be larger than those expected between data sets recorded at different wavelengths, and thus can lead to failure of structure solution.

\section{$7 \mathrm{X}$-ray induced changes in chromophore-containing proteins at $100 \mathrm{~K}$}

The combination of X-ray crystallography with complementary in crystallo spectroscopic techniques, such as optical absorption spectroscopy [57], Raman spectroscopy [58], X-ray absorption spectroscopy [59,60] and electron paramagnetic resonance [61], provided evidence for X-ray induced modifications in crystalline proteins containing chromophores such as metal complexes [62] or conjugated $\pi$-electron systems. Such modifications occur at doses over three orders of magnitude lower than the experimental limit of $30 \mathrm{MGy}$ (see above) determined for global and specific radiation damage at $100 \mathrm{~K}$. For the ferric (oxidized) heme iron in metmyoglobin [62], for instance, at $100 \mathrm{~K} 90 \%$ of the unreduced state remains after a dose (termed spectroscopic lifedose [63]) of 0.01 MGy [63]. Similarly, X-ray induced formation of a spectroscopically observed orange species in the retinal-containing membrane protein bacteriorhodopsin [44] occurs at a lifedose of $0.04 \mathrm{MGy}$ [64]. Other X-ray induced modifications include heme reduction in a photosynthetic reaction center [65], in highmolecular weight cytochrome c [66] and in cytochrome c peroxidase [67], redox changes in a methylamine dehydrogenase [68], deprotonation of the bilin chromophore in a phytochrome [69], and photobleaching of a fluorescent protein [49].

Due to the particularly high radiation sensitivity of chromophore-containing proteins, it is important to identify their redox status by complementary spectroscopic methods when their structures are being solved by X-ray crystallography. As a second step, one or several strategies can be adopted to minimize or even prevent radiation-induced redox modifications (typically reduction). In a composite data-collection strategy, for instance, the X-ray dose is distributed over several locations of a large crystal [70] or over several crystals, as done by Aoyama et al., who collected data from 400 crystals to solve the structure of fully oxidized cytochrome c oxidase [71]. Alternatively, the data collection cryotemperature can be decreased to below the usual $100 \mathrm{~K}$. Indeed, metal reduction has been reported to be reduced 30 -fold when collecting data at $40 \mathrm{~K}$ instead of $110 \mathrm{~K}$ [72]. Additionally, certain scavengers can provide some protection against metal reduction [73].

Although X-ray-induced protein-chromophore reduction is generally a nuisance, it can be put to good use for studying macromolecular processes. A prominent example is the structural characterization of intermediates in the $\mathrm{P} 450$ cam cytochrome reaction pathway, triggered by 
an electron generated by X-ray radiolysis of water [74]. Similarly, several redox intermediates of horseradish peroxidase have been generated by X-ray induced electrons and characterized by the aforementioned dose-dependent composite data-collection strategy [70]. More recently, enzyme catalysis in copper nitrite reductase has been elucidated by serial synchrotron crystallography (SSX) at $100 \mathrm{~K}$ [75] (see below). Furthermore, the catalytic cycle of the non-chromophore-containing urate oxidase, kick-started by X-ray absorption, has been studied by combining in crystallo Raman spectroscopy, QM/MM simulations and X-ray crystallography [76].

\section{Temperature dependence of radiation damage}

Cryocrystallography [2] replaced RT data collection at synchrotron sources some two decades ago [77] because it increased the crystal lifetime in the X-ray beam by up to two orders of magnitude $[8,78,79]$. The main benefit of cryocooling is obtained when decreasing the temperature down to $200 \mathrm{~K}$ [80] where most atomic motions are quenched [81] because of vitrification (transition to a glass state) and dynamical transitions of the solvent and protein, respectively [82-84]. Decreasing the data collection temperature further to $40 \mathrm{~K}$ yields only a very small decrease in specific and global radiation damage compared to $100 \mathrm{~K}[36,85,86]$ (except for the large effect on metal reduction mentioned in the previous section), so that helium cooling has not replaced nitrogen cooling in standard cryocrystallographic experiments.

The combination of X-ray induced structural changes and temperature-controlled crystallography provides a means to initiate and study macromolecular functioning. In the P450cam cytochrome example mentioned above [74], the crystals were transiently thawed to RT after radiolytic electron generation to unlock motional freedom and allow the pathway to proceed. In another example, a substrate analogue bound to crystalline acetylcholinesterase was radiolysed in two dose-dependent data collection series at $100 \mathrm{~K}$ and at $155 \mathrm{~K}$ [87], respectively. Only at $155 \mathrm{~K}$, but not at $100 \mathrm{~K}$, was the protein flexibility high enough so that exit of the radiolytic products could be identified and followed structurally. Temperaturecontrolled crystallography has a long history (reviewed in [88]) and was initiated by Frauenfelder, Petsko and Tsernoglou [89]. If radiation-induced changes are not deliberately incorporated into the experimental protocol as in the two examples above, careful control experiments must be conducted to avoid or deconvolute radiation- from temperature-induced changes, as illustrated by a recent multi-temperature study on crystals of the enzyme cyclophilin A [90]. 


\section{Radiation damage at room temperature}

The first published study of radiation damage in MX was the seminal paper by Blake and Phillips [91] on RT myoglobin crystals. The conclusions drawn were that each $8 \mathrm{keV}$ photon disrupts around 70 protein molecules and disorders a further 90 for doses up to about 0.2 MGy. The $D_{1 / 2}$ was reported as $0.59 \mathrm{MGy}$, around 70 times smaller than the experimental dose limit $D_{1 / 2}$ of 43 MGy [8]. Since the authors observed that the structure factors of some reflections increased slightly, while others decreased, they deduced that specific structural damage to particular amino acid residues must be occurring within the crystal, a hypothesis confirmed many years later [40].

Despite the high rate of damage, RT data collection is currently witnessing a renaissance for several reasons. Technically it has become possible to mount an entire crystallization tray onto a goniometer and irradiate the crystals in situ, with no crystal handling at all [92] and computational tools have been created to enable the images thus collected to be combined into complete datasets. In addition, methods have been developed for enclosing loop-mounted crystals in plastic sleeves [93], which are much less damaging to the crystals than transferring them to quartz or glass capillary tubes. Unenclosed loop-mounted crystals can be protected from drying at RT by special hydration devices [94]. From the standpoint of structural biology, is has now been recognized that the modification of conformational heterogeneity that may occur during cryocooling is avoided in RT experiments [95].

Despite the greatly increased radiation sensitivity at RT compared to $100 \mathrm{~K}$, there is evidence that some of the global radiation damage can be outrun at high dose rates at (or at a lower but close to) RT [79, 96, 97]. Specific damage is very difficult to track in electron density derived from RT measurements, because of the reduced crystal lifetime hampering collection of consecutive dose-dependent datasets, which is possible at $100 \mathrm{~K}$ (see above). Indications of specific damage to disulfide bonds have been reported in traditional oscillation [79] and SSX [98] experiments. However, a more recent SSX study does not report any specific damage, even though the diffraction power of the crystals decreased to $20 \%$ of its original value [30]. The current rapid development of SSX will allow in-depth studies of RT radiation damage because the dose required for collecting a data set is spread over thousands of crystals or more.

10 Practical aspects - wavelength (in)dependence 
The question of whether damage rates depend on the incident X-ray wavelength has been both theoretically and experimentally addressed for MX. Arndt [99] pointed out that both the elastic and photoelectric effect cross sections are greater at lower energy, so in fact the ratio of cross sections for diffraction to absorption events does not change significantly over the incident X-ray energy range used in MX. This was experimentally investigated by Weiss and co-workers [100], who analysed the structures derived from elastase data collected at X-ray energies of 12.4 and $6.2 \mathrm{keV}$, and concluded that there was no difference in rates of specific damage to disulfides and cadmium ions. This result was corroborated by Shimizu and coworkers [101], who collected data at 6.5, 7.1, 8.3, 9.9, 12.4, 16.5, 20.0, 24.8 and $33.0 \mathrm{keV}$ from HEWL crystals, and detected no difference in the rate of specific damage in the corresponding refined structures nor their final atomic B-factors. Moreover, neither were there any significant differences in any of the global damage metrics at the various energies. However, Homer and co-workers [102] found that the rate of electron density decrease (electrons/ $\AA^{3} / \mathrm{MGy}$ ) was greater at $14 \mathrm{keV}$ than at $9 \mathrm{keV}$ for cysteine sulfur atoms involved in disulfide bridges in HEWL crystals, although no statistically significant differences in the decay rates were found for methionine $\mathrm{S}$ atoms. Also, Fourme and co-workers reported an eight-fold increase in data collection efficiency at $33 \mathrm{keV}$ compared with at $8 \mathrm{keV}$ [103]. More recently, Liebschner and co-workers [104] took a series of diffraction images from thaumatin crystals at energies of $6.33,12.66$ and $19.00 \mathrm{keV}$ at $100 \mathrm{~K}$ over small $\left(2^{\circ}\right)$ repetitive rotation intervals and found that for $2.45 \AA$ resolution data, $D_{0.7}$ was $7.5 \mathrm{MGy}$ for the $6.33 \mathrm{keV}$ dataset and for the two higher energies it was $\sim 11 \mathrm{MGy}$. Thus there is as yet no consensus on the question of the dependence of radiation damage rates on incident radiation wavelength.

\section{Practical aspects - is there a dose rate effect at $100 \mathrm{~K}$ ?}

Evidence from experiments with the flux densities currently utilized is that there is no significant dose rate effect at $100 \mathrm{~K}$. Gonzalez and Nave [9] used two different attenuator settings on a $2^{\text {nd }}$ generation synchrotron beamline, and showed similar reflection intensity decay rates. With flux densities of up to $10^{15}$ photons $\mathrm{s}^{-1} \mathrm{~mm}^{-2}$, Sliz and co-workers [105] also detected no dose rate differences in global decay metrics. Another study described similar observations when monitoring global data quality indicators, but on the basis of an analysis of difference electron density maps, concluded that there could be a second-order dose rate effect [106]. A small dose rate dependent $D_{1 / 2}$ decrease was observed by Owen and co- 
workers [28] when monitoring the summed intensity loss. Close to RT, however, significant dose rate effects have been observed (see above).

Under the experimental conditions used in all these studies, crystal heating is not thought to be significant [107]. However with the advent of $4^{\text {th }}$ generation synchrotron beams for MX, this issue should be revisited, since if the beam induces temperature rises above $110 \mathrm{~K}$ in the sample, hydroxyl radicals will become mobile and diffuse through the crystal, thus potentially increasing the rate of damage.

\section{Practical aspects - scavengers}

A possible way to reduce the rate of radiation damage is to make use of small molecule radioprotectants, either by adding them to the cryobuffer prior to flash cooling the crystals, or by soaking the crystals before an RT experiment. These compounds either intercept free radicals (radical scavengers) preventing them from reaching the protein, or repair centres of ionization already present. A number of studies reporting conflicting results have been published, showing significant variation between crystals treated in nominally the same way with scavengers: all (46) different compounds tried prior to 2011 are summarized in a table in the supplementary material of [108]. Some scavengers have been tested by several groups but the results are not always in accord. There is thus a lack of consensus on the efficacy of scavengers that have been investigated by various means. Very few produced more than a twofold increase of $D_{1 / 2}$, which is a criterion suggested by Holton [109] for judging effective radiation damage mitigation strategies.

A number of scavenger studies have observed little, or even adverse effects induced by these additives. For instance, Kmetko and co-workers [110] investigated 19 putative scavengers using the $B_{r e l}$ metric, and found none were effective at $100 \mathrm{~K}$. At RT, they reported that 12 were ineffective, six sensitised the crystals, and only one, sodium nitrate (which is a very efficient electron scavenger), had a protective effect. However, Barker and co-workers [111] found that 1,4-benzoquinone at RT increased the dose tolerance of HEWL crystals to global damage by a factor of nine as monitored by intensity decay, and significantly reduced the specific damage to susceptible residues (with some disulfides remaining undamaged even at $0.62 \mathrm{MGy}$ ). For sodium nitrate at $100 \mathrm{~K}$, de la Mora and co-workers [39] found that specific damage to disulfides was reduced by more than a factor of five compared to the structure determined from an HEWL crystal not soaked in nitrate, while the global damage, as monitored by diffraction intensity decay, was lessened by a factor of two. In that study, in the soaked-crystal structure, a bound nitrate anion adjacent to a disulfide bond was seen to be 
reduced as a function of dose and to significantly protect the bond from cleavage (Figure 5) compared to the non-soaked case. This observation reinforces the evidence that breakage of disulfide bonds is caused by mobile electrons which travel round the protein structure at 100 $\mathrm{K}$ and reduce the most electron-affinic group (in the absence of bound metal cations), and illustrates radiation chemistry in action.

Due to the fundamental disagreement within the MX literature on the general utility of scavengers, they are seldom employed to slow the progression of damage, despite some impressive anecdotal evidence that they can be effective and are worth trying. It should be noted that many components of crystallisation buffers, and in particular cryoprotectants, are already good scavengers for some of the damage agents. For instance, ethylene glycol, PEG and glycerol have high rate constants for scavenging hydroxyl radicals.

\section{What can the experimenter do to minimise radiation damage rates?}

Despite the systematic studies mentioned above, the pivotal question remains: what can the experimenter do to minimise radiation damage rates? There are several general strategies that can make a difference to the damage rate during a data collection. Firstly, if there are any heavy atoms in the buffer (e.g., cacodylate, which contains arsenic), back-soaking can reduce the absorption coefficients of the crystal and thus the rate of damage significantly. Holton [109] used RADDOSE to compute the 'dose doubling concentrations' of various buffers, and for arsenic this is only $350 \mathrm{mM}$. Secondly, matching the beam size to the crystal reduces interactions with the buffer surrounding the crystal and so minimizes the background. Thirdly, if a top-hat beam is available (through, for instance, defocusing the beam and then reducing its size appropriately using slits), this will minimize differential damage across the crystal, and improve the resultant electron density maps. Fourthly, it is worth considering more sophisticated data collection protocols, such as helical scans of rod shaped crystals, composite data set collection, or SSX. Lastly, one should be aware of which parameters might be important in affecting damage rates, and adjust them appropriately, for example, by asking the question “is the highest resolution, or the most complete dataset, my priority?".

\section{Radiation damage in serial femtosecond crystallography at XFELs}

X-ray free electron lasers (XFELs) produce short (several tens of fs) pulses with a peak brilliance 10 orders of magnitude above those of $3^{\text {rd }}$ generation synchrotron sources. They enable data collection before chemical and structural damage has had the time to develop, i.e. recording 'diffraction-before-destruction' [112]. At XFELs, crystallographic data are 
collected in a serial way, called serial femtosecond crystallography (SFX) [113], that leads to high-resolution protein structures [114], in most cases devoid of specific radiation damage $[115,116]$. However, if SFX data are collected at very high doses (e.g. up to 3 GGy [117], but see the cautionary note above on dose calculations for small crystals), global radiation damage has been reported $[117,118]$, and there is evidence that specific damage might occur [117]. Such specific damage has been generated and studied in detail by deliberately exposing ferredoxin crystals to unattenuated 80 fs XFEL pulses at Stanford, leading to an absorbed dose of 30 GGy per crystal [119]. In comparison to a low-dose synchrotron data set, the SFX XFEL data showed reduced electron density for the iron atoms of the two [4Fe-4S] clusters in ferredoxin, with the effect being stronger in one of the clusters. Specific damage at sulfur sites in cathepsin B has been identified in a difference Fourier map computed from SFX data collected with a high and a low X-ray fluence [120]. Consequently, XFEL pulses need to be kept short and attenuated in order to avoid global and specific radiation damage.

\section{Acknowledgments:}

We thank Ian Carmichael and Kathryn Shelley for their comments on this contribution, Charles Bury and Eugenio de la Mora for making the figures, and Markus Gerstel for carrying out a survey of the PDB regarding the temperature of data collection. We also acknowledge those many colleagues with whom we have discussed the issues related to radiation damage in MX. We especially thank the ESRF for access to beamtime since 2000 under the Radiation Damage BAG, which has allowed us to carry out systematic studies on many aspects of the topic. 


\section{FIGURE LEGENDS}

Figure 1. Primary X-ray interaction processes with atoms of the macromolecule and solvent. (a) Elastic (Thomson, coherent) scattering. The waves are phase shifted by $180^{\circ}$ on scattering and add vectorially to give the diffraction pattern. (b) Compton (incoherent) scattering. The $\mathrm{X}$-ray transfers some energy to an atomic electron and thus has lower energy (longer wavelength) after the interaction. Energy is lost in the crystal, contributing to the absorbed dose. (c) Photoelectric absorption. The X-ray transfers all its energy to an atomic electron, which is then ejected and can give rise to the ionization of up to 500 other atoms. The excited atom can then emit a characteristic (fluorescent) X-ray or an Auger electron to return to its ground state.

Figure 2. RADDOSE-3D calculated dose distributions in a TRAP-RNA complex crystal rotated $180^{\circ}$ while irradiated in two different X-ray beams, with (a) a top-hat profile (from EMBL beamline P14, PETRA III, DESY, Hamburg) giving dose values between 18.08 and 18.17 MGy, and (b) a typical Gaussian profile causing a much greater dose inhomogeneity of 0.59 to $112.1 \mathrm{MGy}$.

Figure 3. Three indicators of global radiation damage. (a) Normalised summed intensity decay of complete consecutive datasets for a holoferritin crystal at $100 \mathrm{~K}, I_{n} / I_{i}$, against dose, exhibiting approximately linear decay behaviour (fitted line shown) (b) Relative $B$-factor, $B_{r e l}$ $=B_{n}-B_{1}$ for the same crystal as in (a), showing linear increase (fitted line shown), (c) An idealized plot of $R_{d}$ [38], the pairwise merging $R$-factor ('decay' or 'damage' factor) between identical and symmetry-related reflections occurring on different diffraction images, plotted against the difference in dose, $\Delta D$, between the images on which the reflections were collected, or against the difference in image number if dose values are not available. The plot is a straight line parallel to the $x$ axis if there is no damage, but rises approximately linearly in the presence of damage.

Figure 4. Specific structural damage. (a) Disulfide bond cleavage, (b) Glu decarboxylation, and (c) Met sulphur disordering within myrosinase, PDB: 1DWA [32]. Fobs(4)-Fobs(1) Fourier difference maps between dataset 1 and 4 collected on the same crystal are shown, contoured at +/-5 sigma. Negative difference density (red) indicates disordering of the atomic positions with an accumulated dose of $\sim 14$ MGy. In (a), the positive difference density (green) indicates the repositioning of the two sulfurs upon cleavage of the disulfide bond. The maps were calculated using the phases of the structure refined from dataset 1. 
Figure 5. Radiation chemistry of a scavenger. Dose-dependent nitrate reduction observed in an HEWL crystal soaked in $0.5 \mathrm{M} \mathrm{NaNO}_{3}$ for 4 minutes prior to cryocooling and data collection. The vicinity of the Cys6 - Cys 127 disulfide bond is shown [panels (a)-(f) correspond to structures derived from consecutive datasets $1-6$ at 2.3 to $28.6 \mathrm{MGy}$, respectively]. The 2Fo - Fc map (grey) is contoured at $1.0 \sigma$ and the Fo - Fc map is contoured at $\pm 3.0 \sigma$ (green:+; red: -). A bound nitrate anion is apparently reduced to $\mathrm{NO}_{2}(6.6 \mathrm{MGy})$ and then to NO (23.3 MGy) by mobile electrons produced by the X-ray beam, and the disulfide bond is protected from damage until the nitrate scavenging capacity is exhausted. The refined models corresponding to the PDB codes 2byh, 2byi, 2byj, 2byl, 2bym, 2byn are shown in panels a to f, respectively. 


\section{References}

1. Blundell TL, Johnson LN (1976) Protein Crystallography. Academic Press, London.

2. Hope H (1988) Cryocrystallography of biological macromolecules: a generally applicable method. Acta Crystallogr B44:22-26.

3. Teng T (1990) Mounting of crystals for macromolecular crystallography in a freestanding thin film. J Appl Crystallogr 23:387-391.

4. Rodgers DW (1994) Cryocrystallography. Structure 2:1135-1140.

5. Rodgers DW (1997) Practical cryocrystallography. Methods Enzymol 276:183-203.

6. Garman EF, Schneider TR (1997) Macromolecular cryocrystallography. J Appl Crystallogr 30:211-237.

7. Garman E (1999) Cool data: quantity AND quality. Acta Crystallogr D55:1641-1653.

8. Nave C, Garman EF (2005) Towards an understanding of radiation damage in cryocooled macromolecular crystals. J Synchrotron Radiat 12:257-260. .

9. Gonzalez A, Nave C (1994) Radiation damage in protein crystals at low temperature. Acta Crystallogr D50:874-877.

10. Nave C (1995) Applications of synchrotron X-radiationRadiation damage in protein crystallography. Radiat Phys Chem 45:483-490.

11. Sanishvili R, Yoder DW, Pothineni SB et al. (2011) Radiation damage in protein crystals is reduced with a micron-sized X-ray beam. Proc Natl Acad Sci USA 108:6127-6132.

12. O'Neill P, Stevens DL, Garman EF (2002) Physical and chemical considerations of damage induced in protein crystals by synchrotron radiation: a radiation chemical perspective. J Synchrotron Radiat 9:329-332.

13. Cosier J, Glazer AM (1986) A nitrogen-gas-stream cryostat for general X-ray diffraction studies. J Appl Crystallogr 19:105-107.

14. Owen RL, Axford D, Nettleship JE et al. (2012) Outrunning free radicals in roomtemperature macromolecular crystallography. Acta Crystallogr D68:810-818.

15. Jones GD, Lea JS, Symons MC et al (1987) Structure and mobility of electron gain and loss centres in proteins. Nature 330:772-773.

16. Owen RL, Holton JM, Schulze-Briese C et al (2009) Determination of X-ray flux using silicon pin diodes. J Synchrotron Radiat. 16:143-151.

17. Zeldin OB, Gerstel M, Garman EF (2013) RADDOSE-3D: time- and space-resolved modelling of dose in macromolecular crystallography. J Appl Crystallogr 46:12251230.

18. Zeldin OB, Gerstel M, Garman EF (2013) Optimizing the spatial distribution of dose in X-ray macromolecular crystallography. J Synchrotron Radiat 20:49-57.

19. Flot D, Mairs T, Giraud T et al. (2010) The ID23-2 structural biology microfocus beamline at the ESRF. J Synchrotron Radiat 17:107-118.

20. Cowan JA, Nave C (2008) The optimum conditions to collect X-ray data from very small samples. J Synchrotron Radiat 15:458-462.

21. Murray JW, Garman EF, Ravelli RBG (2004) X-ray absorption by macromolecular crystals: the effects of wavelength and crystal composition on absorbed dose. J Appl Crystallogr 37:513-522.

22. Paithankar KS, Owen RL, Garman EF (2009) Absorbed dose calculations for macromolecular crystals: improvements to RADDOSE. J Synchrotron Radiat. 16:152162.

23. Paithankar KS, Garman EF (2010) Know your dose: RADDOSE. Acta Crystallogr D66:381-388. 
24. Krojer T, von Delft F (2011) Assessment of radiation damage behaviour in a large collection of empirically optimized datasets highlights the importance of unmeasured complicating effects. J Synchrotron Radiat 18:387-397.

25. Zeldin OB, Brockhauser S, Bremridge J et al (2013) Predicting the X-ray lifetime of protein crystals. Proc Natl Acad Sci USA 110:20551-20556.

26. Henderson R (1990) Cryo-protection of protein crystals against radiation damage in electron and X-ray diffraction. Proc R Soc Lond B 241:6-8.

27. Murray JW, Rudino-Pinera E, Owen RL et al. (2005) Parameters affecting the X-ray dose absorbed by macromolecular crystals. J Synchrotron Radiat 12:268-275.

28. Owen RL, Rudino-Pinera E, Garman EF (2006) Experimental determination of the radiation dose limit for cryocooled protein crystals. Proc Natl Acad Sci USA 103:4912-4917.

29. Howells MR, Beetz T, Chapman HN et al. (2009) An assessment of the resolution limitation due to radiation-damage in $\mathrm{x}$-ray diffraction microscopy. J Electron Spectrose 170:4-12.

30. Roedig P, Duman R, Sanchez-Weathertby J et al. (2016) Room-temperature macromolecular crystallography using a micro-patterned silicon chip with minimal background scattering. J Appl Crystallogr 49:968-975.

31. Ravelli RB, McSweeney SM (2000) The 'fingerprint' that X-rays can leave on structures. Structure 8:315-328.

32. Burmeister WP (2000) Structural changes in a cryo-cooled protein crystal owing to radiation damage. Acta Crystallogr D56:328-341.

33. Teng T, Moffat K (2000) Primary radiation damage of protein crystals by intense synchrotron radiation. J. Synchrotron Radiat 7:313-317.

34. Murray J, Garman E (2002) Investigation of possible free-radical scavengers and metrics for radiation damage in protein cryocrystallography. J Synchrotron Radiat 9:347-354.

35. Ravelli RB, Theveneau P, McSweeney S et al (2002) Unit-cell volume change as a metric of radiation damage in crystals of macromolecules. J Synchrotron Radiat 9:355-360.

36. Meents A, Gutmann S, Wagner A et al (2010) Origin and temperature dependence of radiation damage in biological samples at cryogenic temperatures. Proc Natl Acad Sci USA 107:1094-1099.

37. Kmetko J, Husseini NS, Naides M et al (2006) Quantifying X-ray radiation damage in protein crystals at cryogenic temperatures. Acta Crystallogr D62:1030-1038.

38. Diederichs K, McSweeney S, Ravelli RB (2003) Zero-dose extrapolation as part of macromolecular synchrotron data reduction. Acta Crystallogr D59:903-909.

39. De la Mora E, Carmichael I, Garman EF (2011) Effective scavenging at cryotemperatures: further increasing the dose tolerance of protein crystals. J Synchrotron Radiat 18:346-357.

40. Helliwell JR (1988) Protein crystal perfection and the nature of radiation damage. J Cryst Growth 90:259-272.

41. Weik M, Ravelli RB, Kryger G et al (2000) Specific chemical and structural damage to proteins produced by synchrotron radiation. Proc Natl Acad Sci USA 97:623-628.

42. Weik M, Berges J, Raves ML et al (2002) Evidence for the formation of disulfide radicals in protein crystals upon X-ray irradiation. J Synchrotron Radiat 9:342-346.

43. Sutton KA, Black PJ, Mercer KR et al (2013) Insights into the mechanism of X-rayinduced disulfide-bond cleavage in lysozyme crystals based on EPR, optical absorption and X-ray diffraction studies. Acta Crystallogr D69:2381-2394. 
44. Matsui Y, Sakai K, Murakami M et al (2002) Specific damage induced by X-ray radiatioin and structural changes in the primary photoreaction of bacteriorhodopsin. $\mathbf{J}$ Mol Biol 324:469-481.

45. Kort R, Hellingwerf KJ, Ravelli RB (2004) Initial events in the photocycle of photoactive yellow protein. J Biol Chem 279:26417-26424.

46. Mees A, Klar T, Gnau P et al (2004) Crystal structure of a photolyase bound to a CPD-like DNA lesion after in situ repair. Science 306:1789-1793.

47. Fioravanti E, Vellieux FM, Amara P, et al (2007) Specific radiation damage to acidic residues and its relation to their chemical and structural environment. J Synchrotron Radiat 14:84-91.

48. Sjoblom B, Polentarutti M, Djinovic-Carugo K (2009) Structural study of X-ray induced activation of carbonic anhydrase. Proc Natl Acad Sci USA. 106:10609-10613.

49. Adam V, Carpentier P, Violot S et al (2009) Structural basis of X-ray-induced transient photobleaching in a photoactivatable green fluorescent protein. J Am Chem Soc 131:18063-18065.

50. Dubnovitsky AP, Ravelli RB, Popov AN et al (2005) Strain relief at the active site of phosphoserine aminotransferase induced by radiation damage. Protein Sci 14:14981507.

51. Bury C, Garman EF, Ginn HM et al. (2015) Radiation damage to nucleoprotein complexes in macromolecular crystallography. J Synchrotron Radiat 22:213-224.

52. Bury CS, McGeehan JE, Antson AA et al. (2016) RNA protects a nucleoprotein complex against radiation damage. Acta Crystallogr D72:648-657.

53. Ravelli RB, Leiros HK, Pan B et al (2003) Specific radiation damage can be used to solve macromolecular crystal structures. Structure 11:217-224.

54. Banumathi S, Zwart PH, Ramagopal UA et al (2004) Structural effects of radiation damage and its potential for phasing. Acta Crystallogr D60:1085-1093.

55. Ravelli RB, Nanao MH, Lovering A et al (2005) Phasing in the presence of radiation damage. J Synchrotron Radiat 12:276-284.

56. Nanao MH, Ravelli RB (2006) Phasing macromolecular structures with UV-induced structural changes. Structure 14:791-800.

57. McGeehan J, Ravelli RGB, Murray JW et al (2009) Colouring cryo-cooled crystals: online microspectrophotometry. J Synchrotron Radiat 16:163-172.

58. Carpentier P, Royant A, Ohana J et al D (2007) Advances in spectroscopic methods for biological crystals. 2. Raman spectroscopy. J Appl Crystallogr 40:1113-1122.

59. Yano J, Kern J, Irrgang K-D et al. (2005) X-ray damage to the Mn4Ca complex in single crystals of photosystem II: a case study for metalloprotein crystallography. Proc Natl Acad Sci USA. 102:12047-12052.

60. Holton JM (2007) XANES measurements of the rate of radiation damage to selenomethionine side chains. J Synchrotron Radiat 14:51-72.

61. Utschig LM, Chemerisov SD, Tiede DM et al (2008) Electron paramagnetic resonance study of radiation damage in photosynthetic reaction center crystals. Biochemistry 47:9251-9257.

62. Beitlich T, Kuhnel K, Schulze-Briese C et al (2007) Cryoradiolytic reduction of crystalline heme proteins: analysis by UV-Vis spectroscopy and X-ray crystallography. J Synchrotron Radiat 14:11-23.

63. Hersleth HP, Andersson KK (2011) How different oxidation states of crystalline myoglobin are influenced by X-rays. Biochim Biophys Acta 1814:785-796.

64. Borshchevskiy V, Round E, Erofeev I et al (2014) Low-dose X-ray radiation induces structural alterations in proteins. Acta Crystallogr D70:2675-2685. 
65. Baxter RH, Seagle BL, Ponomarenko N et al (2004) Specific radiation damage illustrates light-induced structural changes in the photosynthetic reaction center. J Am Chem Soc 126:16728-16729.

66. Sato M, Shibata N, Morimoto Y et al. (2004) X-ray induced reduction of the crystal of high-molecular-weight cytochrome c revealed by microspectrophotometry. J Synchrotron Radiat 11:113-116.

67. Echalier A, Goodhew CF, Pettigrew GW et al (2006) Activation and catalysis of the di-heme cytochrome c peroxidase from Paracoccus pantotrophus. Structure 14:107117.

68. Pearson AR, Pahl R, Kovaleva EG et al (2007) Tracking X-ray-derived redox changes in crystals of a methylamine dehydrogenase/amicyanin complex using single-crystal UV/Vis microspectrophotometry. J Synchrotron Radiat 14:92-98.

69. Li F, Burgie ES, Yu T et al (2015) X-ray radiation induces deprotonation of the bilin chromophore in crystalline D. radiodurans phytochrome. J Am Chem Soc 137:27922795.

70. Berglund GI, Carlsson GH, Smith AT et al (2002) The catalytic pathway of horseradish peroxidase at high resolution. Nature 417:463-468.

71. Aoyama H, Muramoto K, Shinzawa-Itoh K et al (2009) A peroxide bridge between $\mathrm{Fe}$ and $\mathrm{Cu}$ ions in the $\mathrm{O} 2$ reduction site of fully oxidized cytochrome $\mathrm{c}$ oxidase could suppress the proton pump. Proc Natl Acad Sci USA 106:2165-2169.

72. Corbett MC, Latimer MJ, Poulos TL et al (2007) Photoreduction of the active site of the metalloprotein putidaredoxin by synchrotron radiation. Acta Crystallogr D63:951960.

73. Macedo S, Pechlaner M, Schmid W et al (2009) Can soaked-in scavengers protect metalloprotein active sites from reduction during data collection? J Synchrotron Radiat 16:191-204. .

74. Schlichting I, Berendzen J, Chu K et al (2000) The catalytic pathway of cytochrome p450cam at atomic resolution. Science 287:1615-1622.

75. Horrell S, Antonyuk SV, Eady RR et al (2016) Serial crystallography captures enzyme catalysis in copper nitrite reductase at atomic resolution from one crystal. IUCr $\mathbf{J}$ 3:271-281.

76. Bui S, von Stetten D, Jambrina PG et al (2014) Direct evidence for a peroxide intermediate and a reactive enzyme-substrate-dioxygen configuration in a cofactorfree oxidase. Angew Chem Int Ed 53:13710-13714.

77. Garman EF, Doublie S (2003) Cryocooling of macromolecular crystals: optimization methods. Methods Enzymol 368:188-216.

78. Southworth-Davies RJ, Medina MA, Carmichael I et al (2007) Observation of decreased radiation damage at higher dose rates in room temperature protein crystallography. Structure 15:1531-1541.

79. Warkentin M, Hopkins JB, Badeau R et al (2013) Global radiation damage: temperature dependence, time dependence and how to outrun it. J Synchrotron Radiat 20:7-13.

80. Warkentin M, Thorne RE (2010) Glass transition in thaumatin crystals revealed through temperature-dependent radiation-sensitivity measurements. Acta Crystallogr D66:1092-1100.

81. Halle B (2004) Biomolecular cryocrystallography: Structural changes during flashcooling. Proc Natl Acad Sci USA 101:4793-4798.

82. Vitkup D, Ringe D, Petsko GA et al (2000) Solvent mobility and the protein 'glass' transition. Nat Struct Biol 7:34-38. 
83. Weik M, Kryger G, Schreurs AM et al (2001) Solvent behaviour in flash-cooled protein crystals at cryogenic temperatures. Acta Crystallogr D 57:566-573.

84. Schiro G, Fichou Y, Gaillat F-X et al (2015) Translational diffusion of hydration water correlates with functional motions in folded and intrinsically disordered proteins. Nature Commun 6:6490.

85. Chinte U, Shah B, Chen Y-S et al (2007) Cryogenic ( $<20 \mathrm{~K})$ helium cooling mitigates radiation damage to protein crystals. Acta Crystallogr D63:486-492..

86. Meents A, Wagner A, Schneider R et al (2007) Reduction of X-ray-induced radiation damage of macromolecular crystals by data collection at $15 \mathrm{~K}$ : a systematic study. Acta Crystallogr D63:302-309.

87. Colletier JP, Bourgeois D, Sanson B et al (2008) Shoot-and-Trap: use of specific X-ray damage to study structural protein dynamics by temperature-controlled cryocrystallography. Proc Natl Acad Sci USA 105:11742-11747.

88. Weik M, Colletier J-P (2010) Temperature-dependent macromolecular X-ray crystallography. Acta Crystallographica D66:437-446.

89. Frauenfelder H, Petsko GA, Tsernoglou D (1979) Temperature-dependent X-ray diffraction as a probe of protein structural dynamics. Nature 280:558-563.

90. Keedy DA, Kenner LR, Warkentin M et al (2015) Mapping the conformational landscape of a dynamic enzyme by multitemperature and XFEL crystallography. eLife 4:e07574.

91. Blake C, Phillips DC (1962) Effects of X-irradiation on single crystals of myoglobin. In: Proceedings of the Symposium on the Biological Effects of Ionising Radiation at the Molecular Level. Vienna, pp. 183-191.

92. Jacquamet L, Ohana J, Joly J et al (2004) Automated analysis of vapor diffusion crystallization drops with an X-ray beam. Structure 12:1219-1225.

93. Kalinin Y, Kmetko J, Bartnik A et al (2005) A new sample mounting technique for room-temperature macromolecular crystallography. J Appl Crystallogr 38:333-339.

94. Sanchez-Weatherby J, Bowler MW, Huet J et al (2009) Improving diffraction by humidity control: a novel device compatible with X-ray beamlines. Acta Crystallogr D65:1237-1246.

95. Fraser JS, van den Bedem H, Samelson AJ et al (2011) Accessing protein conformational ensembles using room-temperature X-ray crystallography. Proc Natl Acad Sci USA 108:16247-16252.

96. Warkentin M, Badeau R, Hopkins JB et al (2012) Global radiation damage at 300 and $260 \mathrm{~K}$ with dose rates approaching $1 \mathrm{MGy} \mathrm{s}^{-1}$. Acta Crystallogr D68:124-133.

97. Owen RL, Paterson N, Axford D et al (2014) Exploiting fast detectors to enter a new dimension in room-temperature crystallography. Acta Crystallogr D70:1248-1256.

98. Coquelle N, Brewster AS, Kapp U et al (2015) Raster-scanning serial protein crystallography using micro- and nano-focused synchrotron beams. Acta Crystallogr D71:1184-1196.

99. Arndt UW (1984) Optimum X-ray wavelength for protein crystallography. J Appl Crystallogr 17:118-119.

100. Weiss MS, Panjikar S, Mueller-Dieckmann C et al (2005) On the influence of the incident photon energy on the radiation damage in crystalline biological samples. $\mathrm{J}$ Synchrotron Radiat 12:304-309.

101. Shimizu N, Hirata K, Hasegawa K et al (2007) Dose dependence of radiation damage for protein crystals studied at various X-ray energies. J Synchrotron Radiat 14:4-10.

102. Homer C, Cooper L, Gonzalez A (2011) Energy dependence of site-specific radiation damage in protein crystals. J Synchrotron Radiat 18:338-345. 
103. Fourme R, Honkimäki V, Girard E et al (2012) Reduction of radiation damage and other benefits of short wavelengths for macromolecular crystallography data collection. J Appl Crystallogr 45:652-661.

104. Liebschner D, Rosenbaum G, Dauter M et al (2015) Radiation decay of thaumatin crystals at three X-ray energies. Acta Crystallogr D 71:772-778.

105. Sliz P, Harrison SC, Rosenbaum G (2003) How does radiation damage in protein crystals depend on X-ray dose? Structure 11:13-19.

106. Leiros HK, Timmins J, Ravelli RB et al (2006) Is radiation damage dependent on the dose rate used during macromolecular crystallography data collection? Acta Crystallogr D62:125-132.

107. Mhaisekar A, Kazmierczak MJ, Banerjee R (2005) Three-dimensional numerical analysis of convection and conduction cooling of spherical biocrystals with localized heating from synchrotron X-ray beams. J Synchrotron Radiat 12:318-328.

108. Allan EG, Kander MC, Carmichael I et al (2013) To scavenge or not to scavenge, that is STILL the question. J Synchrotron Radiat 20:23-36.

109. Holton JM (2009) A beginner's guide to radiation damage. J Synchrotron Radiat 16:133-142.

110. Kmetko J, Warkentin M, Englich U et al (2011) Can radiation damage to protein crystals be reduced using small-molecule compounds? Acta Crystallogr D67:881-893.

111. Barker AI, Southworth-Davies RJ, Paithankar KS et al (2009) Room-temperature scavengers for macromolecular crystallography: increased lifetimes and modified dose dependence of the intensity decay. J Synchrotron Radiat 16:205-216.

112. Neutze R, Wouts R, van der Spoel D et al (2000) Potential for biomolecular imaging with femtosecond X-ray pulses. Nature 406:752-757.

113. Chapman HN, Fromme P, Barty A et al (2011) Femtosecond X-ray protein nanocrystallography. Nature 470:73-77.

114. Boutet S, Lomb L, Williams GJ et al (2012) High-resolution protein structure determination by serial femtosecond crystallography. Science 337:362-364.

115. Hirata K, Shinzawa-Itoh K, Yano N et al (2014) Determination of damage-free crystal structure of an X-ray-sensitive protein using an XFEL. Nature Methods 11:734-736.

116. Chreifi G, Baxter EL, Doukov T et al (2016) Crystal structure of the pristine peroxidase ferryl center and its relevance to proton-coupled electron transfer. Proc Natl Acad Sci USA 113:1226-1231.

117. Lomb L, Barends TRM, Kassemeyer $\mathrm{S}$ et al (2011) Radiation damage in protein serial femtosecond crystallography using an X-ray free-electron laser. Phys Rev B84:214111.

118. Barty A, Caleman C, Aquila A et al (2012) Self-terminating diffraction gates femtosecond X-ray nanocrystallography measurements. Nat Photonics 6:35-40.

119. Nass K, Foucar L, Barends TR et al (2015) Indications of radiation damage in ferredoxin microcrystals using high-intensity X-FEL beams. J Synchrotron Radiat 22:225-238.

120. Galli L, Son S-K, Klinge M et al (2015) Electronic damage in S atoms in a native protein crystal induced by an intense X-ray free-electron laser pulse. Struct Dyn 2:041703. 